\title{
¿Qué hay de nuevo, radio? Claves para un análisis sobre el futuro de la radio española desde el constructivismo social de la tecnología
}

Montse Bonet,Unviersitat Autònoma de Barcelona- montse.bonet@uab.cat

\section{Resumen}

Las claves de lo que será la radio española en el futuro hunden sus raíces en el pasado y dependen en buena medida de la capacidad de transformar ese pasado en una ventaja más que en un lastre. Este artículo repasa lo más destacado de las últimas dos décadas de la radio española para determinar qué es nuevo y qué no lo es, con el fin de no dejar su futuro en manos de profecías autocumplidas. En el marco de las aproximaciones teóricas conocidas globalmente como constructivismo social de la tecnología y a partir de cinco parámetros de estudio (que incluye marco legal, tecnología, programación, financiación y estructura empresarial) el artículo reflexiona sobre los colectivos sociales en manos de los cuales está el futuro del medio.

\section{Palabras clave}

Radio, España, constructivismo social, path-dependence, tecnología.

\section{Abstract}

The keys of what Spanish radio will be in the future are rooted in the past and depend largely on the ability to transform this past into a benefit rather than a burden. This article reviews the highlights of the last two decades of the Spanish radio to determine what is new and what is not, in order not to leave its future in the hands of self-fulfilling prophecies. In the context of theoretical approaches known as social constructivism of technology (SCOT) and from a five-parameter study (including legal framework, technology, programming, financing and corporate structure) the article reflects on social groups in whose hands lies the future of this medium.

Keywords

Radio, Spain, social constructivism, path-dependence, technology.

Sumario

1. Introducción. 2. Una historia no lineal. 3. Tecnología y cadena de valor: radio reticular. 4. Marco normativo: arquitectura digital sobre papel mojado. 5. Estructura empresarial y programación. 6. Financiación. 7. Discusión y conclusiones. 8. Bibliografía. 


\section{Introducción}

La radio española del futuro se está construyendo ahora mismo, experimentando día a día pero con algunos condicionantes del pasado. Es lo que suele conocerse como path dependence (Liebowitz and Margolis, 2000; Mahoney, 2000; Page, 2006; David, 2007), un término de gran complejidad epistemológica cuyo significado suele reducirse al history matters, es decir, la historia importa y tiene un peso específico que extiende su influencia sobre el presente. Sin embargo, no debe tomarse como una relación determinista sino como una herencia recibida que influye en el momento de tomar decisiones, redactar nuevas políticas o cambiar las reglas del juego (Voltmer, 2013). Los cambios técnicos tienen también su path-dependence (MacKenzie y Wajcman, 1999: 19-20): «Which of two or more technologies eventually succeed is not determined by their intrinsic characteristics alone, but also by their histories of adoption. (...) Path-dependence means that local, short-term contingencies can exercise lasting effects».

Este artículo repasa lo más destacado de las últimas dos décadas de la radio española para determinar qué es nuevo y qué no lo es, para no dejar su futuro en manos de profecías autocumplidas. Abordaremos el análisis a partir de cinco parámetros que han resultado de gran utilidad para el estudio del medio en anteriores análisis: marco legal, tecnología, programación, financiación y estructura empresarial; entendiendo que todos se interrelacionan estrechamente. Con ello, podrá observarse en manos de quién está el futuro del medio. A tal fin, nuestro marco teórico se armará a partir de las aportaciones más relevantes de los principales autores que representan la aproximación teórica conocida como construcción social de la tecnología (SCOT, en sus siglas en inglés), que son los que mejor permiten entender que la radio es y será lo que colectivamente queramos que sea.

\section{Una historia no lineal}

El constructivismo social de la tecnología nació como antídoto al determinismo y la idea del desarrollo autónomo de la tecnología (Aibar Puente, 2001), y bebió de los principios metodológicos de la sociología del conocimiento científico (SKK, Social Scientific Knowledge) (Williams y Edge, 1996; Tosoni, 2016). Dentro del constructivismo, diversos autores y corrientes se entrecruzan y colaboran pero todos tienen un objetivo común: desterrar la idea reduccionista y unidireccional de que la tecnología influye sobre el devenir histórico sin que haya vía de retorno ni pueda modificarse dicha evolución.

Los principales representantes y padres fundadores del constructivismo aplicado a la tecnología (en general, no solamente a las TIC, Tecnologías de la Información y la Comunicación) fueron Wiebe Bijker (1995, 2012), Trevor Pinch (1999 y co-editor en Bijker, 2012), Donald Mackenzie y Judy Wajcman (editores, 1999), Thomas P. Hugues (1989), y Michel Callon (en la obra colectiva de Bijker y Pinch, 2012). Podríamos añadir las interesantes aportaciones de Brian Winston (1998), centradas en los medios, e incluso la obra clave de Rogers (2003) sobre la difusión de la innovación o al teórico, activista y siempre lúcido Langdon Winner (1989).

A pesar de que como objeto de estudio la radio no suele interesar demasiado (Piñeiro Otero, 2015)1, algunos autores han puesto el foco en su estudio desde premisas constructivistas. Tal sería el caso, por ejemplo, de Ala-Fossi (2005) analizando el estándar de audio digital DAB (Digital Audio Broadcasting), o el mismo autor y otros (2008 y 2010) en varios estudios comparados de la radio digital en distintos países. Campostrini (2015) basa toda su proyecto de final de carrera en la aplicación del social construction of technology (SCOT) a la radio digital, utilizando para ello la aplicación directa de dos de las mayores aportaciones teóricas y metodológicas del SCOT: la «flexibilidad interpretativa» y los «grupos sociales relevantes», es decir, las diferentes formas en las que las tecnologías son diseñadas pero también entendidas e interpretadas por el público en general, y los grupos sociales de todo tipo que quedan afectados, para bien o para mal, por una tecnología. El por qué son relevantes es uno de los puntos sobre el cual los representantes del SCOT recibieron más críticas.

Finalmente, podríamos añadir algunos trabajos más recientes, de carácter divulgativo en algún caso, estructurados bajo los mismos principios constructivistas, como el manual de Fernández-Quijada (2011) que incluye dos capítulos dedicados al audio, o la investigación sobre innovación tecnológica y servicio público audiovisual (Bonet et al., 2012).

En el caso concreto de la radio, el constructivismo ha resultado un marco teórico de gran utilidad para dar cuenta del éxito o fracaso, según el país, de la radio digital. Pero no es la primera vez que se utiliza, de hecho Winston (1998) dedica un capítulo entero al análisis de la radio desde su nacimiento como medio de comunicación punto a punto hasta convertirse en medio de masas. Dos de sus principales ideas, al analizar el proceso mediante el cual un prototipo entra en el mercado y se convierte en invención, son la presencia o ausencia de una necesidad social sobrevenida (supervening social necessity) y lo que él llama ley de supresión del potencial radical (law of the suppresion of radical potential), en definitiva lo que actúa como acelerador o como freno en el desarrollo de una tecnología y propicia o pone trabas a su entrada en el mercado y su aceptación social.

Fijémonos en cómo todas las ideas fundamentales contenidas en el constructivismo social se nos ofrecen como útiles herramientas de interpretación de muchas de las cosas vividas por la radio, española o mundial, durante su historia. Los grupos sociales afectados por ella, las formas distintas de entenderla, las necesidades sociales que fomentaron su uso fuera del ámbito militar o comercial y los escollos superados. El constructivismo es holístico y por ello incluye no solamente las tecnologías de éxito sino también las fallidas. Pero, más allá de su dimensión tecnológica, la radio es una forma de diseminación de contenidos culturales con carga simbólica y por ello resulta también interesante recuperar la idea de Hugues (1989) de los sistemas tecnológicos y su complejidad, que él define como formados por «messy, complex, problem-solving components. They are both socially constructed and society shaping» (p.51). En un sistema tecnológico, según el autor, tienen cabida artefactos físicos, organizaciones (bancos, empresas), elementos científicos (libros, artículos, investigación universitaria), legislativos (leyes y normas de todo tipo ) y recursos naturales. Hasta el punto de que la combinación de todo ello constituye un «estilo tecnológico» propio y distintivo.

Muchos de estos autores no escribieron sobre radio pero sus reflexiones y análisis son de gran aplicabilidad a nuestro objeto de estudio.

${ }^{1}$ Totalmente cierto, a pesar de que este artículo citado solamente se refiere a la producción en revistas españolas. 
Finalmente, analizando un medio tan normativizado como la radio, no podemos sustraernos a la tentación de preguntarnos, igual que Winner (1999), si los artefactos técnicos tienen cualidades políticas².

Como comentábamos al inicio de este artículo, el análisis del medio se hará a partir de cinco parámetros. Pero aún entendiendo que todos se interrelacionan, empezaremos por el que en los últimos años se ha considerado el factor de mayor influencia sobre el desarrollo de la radio: la tecnología. De entre las industrias culturales, las de flujo (radio y televisión) no han sufrido de momento tanto como las de stock o de edición discontínua (cine, industria musical) o las de difusión discontínua (prensa) a causa de la digitalización y la irrupción de internet en sus cadenas de valor. Sin dudar de que la digitalización e internet han llegado para quedarse, no es menos cierto que los cambios augurados hace veinte años 0 más se producen a un ritmo que nada tiene que ver con la velocidad anunciada y deseada por algunos gurús, políticos y empresarios. La televisión ha notado y mucho la presión del sector de las telecomunicaciones y la telefonía móvil para conseguir parte del espectro ocupado por ella casi en exclusiva durante casi 70 años. No por otro motivo se produjo el apagón digital y se subastó el dividendo digital, es decir, las frecuencias que liberó en aquel proceso en la banda de $800 \mathrm{MHz}$. (Bonet et al., 2008; Bonet y Guimerà, 2016). La radio, por el momento, no se ha visto afectada apenas pues las frecuencias más codiciadas son las de la UHF pero, tratándose de espectro radioeléctrico, no puede darse nada por seguro.

\section{Tecnología y cadena de valor: radio reticular}

Radio y tecnología son inseparables y el desarrollo de una no se entiende sin el devenir de la otra. Esta estrecha relación suele generar toda suerte de discursos de raíz determinista al asegurar que el avance tecnológico es imparable y nada puede detenerlo, no cabe, pues, sino abrazarlo y asumirlo. En España, casos como el del DAB han contribuído a demostrar que el SCOT es algo más que un conjunto de aproximaciones teóricas defendidas por ingenieros o sociológos, sirviendo como ejemplo de cómo la combinación de distintos factores y el rechazo de varios colectivos sociales llevaron a una buena tecnología (en cuanto a calidad técnica) al limbo.

Aunque el determinismo tecnológico ha existido siempre, hay un punto de inflexión en el que su contenido casi se convirtió en dogma y ese momento fue el nacimiento de la Sociedad de la Información como proyecto político y económico, aupado por la digitalización. Efectivamente, conocidos son ya los antecedentes ideológicos en obras como la de Daniel Bell y su sociedad post-industrial (1972), el Informe Nora-Minc (1980, original de 1978), el Informe Jacudi (Japan Computer Usage Development Institute, 1980, original de 1972) y su traducción material en las National Information Infrastructure (NII) de Bill Clinton y Al Gore a inicios de los 90.

Este discurso, convenientemente aderezado con los aires desreguladores de los años 80, convirtió las TIC en la piedra angular del desarrollo social, económico y de crecimiento del mundo. Y ya no hubo vuelta atrás. Determinar que el conocimiento y la cultura serán fuente de riqueza convirtió las TIC pero también la cultura y la comunicación en general en elementos contables. Aunque en menor medida, la radio ha visto su cadena de valor atravesada por la digitalización y la aparición de una miríada de alternativas que amenazan su statu quo, al igual que lo hizo en su momento el DAB, aunque no por los mismos motivos (Bonet et al., 2009). Por la parte de la radio digital (DAB), la amenaza ha quedado detenida de momento pero internet y la universalización tecnológica la han obligado a cuestionarse su core business y de qué manera debe rediseñarse su cadena de valor.

Las múltiples definiciones de cadena de valor suelen usar como base la clásica de Porter (1998) si bien en los últimos años se ha ido ampliando al verse cruzada transversalmente por la digitalización. Una cadena de valor de contexto digital aumenta su complejidad:

a) al permitir intercambiar los roles entre productores y consumidores;

b) al contar con más productores no tradicionales (y ni siquiera «alternativos» como podrían ser hasta ahora las radios culturales, piratas 0 comunitarias);

c) al introducir mayor número puntos de difusión en forma de plataformas tecnológicas o dispositivos electrónicos que, al mismo tiempo,

d) enriquecen las formas de consumo;

e) al permitir el desglose de la clásica parrilla programática y, consecuentemente,

f) al modificar la forma de producción convirtiendo el producto de flujo en productos de catálogo, creando un stock;

g) al poner en cuestión las rutinas de producción y los perfiles profesionales;

h) y vuelta a empezar desde el principio, pues la digitalización permite una distribución reticular de la producción y difusión de contenidos.

Aunque todos estos puntos existen y se están experimentando en mayor o menor grado, su desarrollo no ha sido ni está siendo tan rápido ni tan planificado como algunos voceros de la Sociedad de la Información hubieran querido y mucho menos para el caso de un medio del que muchos opinan pero pocos estudian. Los contenidos de audio y la industria radiofónica están mutando pero en España todavía hay factores que siguen anclando el medio en el pasado. «Ens trobem davant d'un nou context en què convergeixen la lògica dels mitjans tradicionals i noves pràctiques comunicatives d'uns ciutadans que intervenen en el flux de la informació i interactuen amb altres individus i amb els mitjans» (Sellas, 2013: 130). Esta complejidad y la presencia activa de más actores pone en cuestión e incluso democratiza el papel del mediador y, por ende, el del gatekeeper.

\section{Marco normativo: arquitectura digital sobre papel mojado}

A nivel europeo, la radio es considerada un negocio local (referido al nivel estatal) por lo que no cabe transponer normas ni directivas, como sí hay que cumplir en el caso de la televisión. La radio en España sigue estructurada en un sistema de tres capas (estatal, autonómico y local), donde conviven radios públicas y privadas, financiándose casi todas con publicidad (menos RNE desde 1993). La democracia puso cierto orden jurídico al sector, de forma bastante dispersa y sin plantear nunca una reforma integral, de conjunto. La Ley General de la Comunicación Audiovisual de 2010 puso fin a la dispersión normativa pero afianzó «un modelo de corte neoliberal que define la radio como un servicio de interés general, muy lejos del concepto de servicio público vigente desde los orígenes del medio» (Arboledas, 2012:138).

2 En referencia a su famoso escrito «Do artifacts have politics?«, incluido en el libro editado por Donald MacKenzie y Judy Wajcman (1999) The social shaping of technology. 
Nada de esto se entendería si no se indaga en las causas profundas, que no son otras que el mantenimiento de una estructura subyacente que no ha perdido las características que definieron a esta industria cultural durante el franquismo. La transición trajo consigo nuevas voces, renovación tecnológica, mayor seguridad jurídica y modernización de las formas de gestión, así como libertad de expresión y nuevos géneros. Pero todo ello, que terminó nuevamente en oligopolio y concentración (Bonet, 2005), se construyó sobre un sistema político clientelar, que durante décadas utilizó el sistema concesional como moneda de cambio (Arboledas y Bonet, 2014; Bonet, Arboledas y Guimerà, 2014). La Ley General de Comunicación Audiovisual supuso el paso de la concesión administrativa a la licencia, confinando en exclusiva a la radio de servicio público al sector de los medios financiados con dinero público.

El paso de la dictadura a la democracia en España, citado a menudo como ejemplo de transición pacífica, ocurrió al tiempo que en Europa empezaban a soplar aires desreguladores de corte neo-liberal. La transición de los medios de comunicación españoles resultó en una mezcla de libertad de expresión y apuesta por la democracia en unas emisoras de radio y una televisión fuertemente instrumentalizados políticamente. El control, como decíamos, se ejerce a través de la concesión o licencia, en el caso de la radio privada, pero en cuanto a las radios públicas, tengan la cobertura que tengan, se controlan a través de leyes que se aseguran de que el componente profesional y social no pueda estar nunca por encima del político 0, siendo más exactos, no pueda resultar partidista. La creación en 2004 del Consejo Independiente de Reforma de los Medios Públicos de Comunicación, conocido como «comité de sabios», a instancias del entonces presidente del gobierno, José Luís Rodríguez Zapatero, fue un intento serio y valiente (para los cánones al uso en España) y sirvió de base para la redacción de la Ley 17/2006, de 5 de junio, de la radio y la televisión de titularidad estatal. Tanto las conclusiones del informe como buena parte de la Ley fueron bastante bien acogidos por la profesión periodística que vio en ellos un primer intento serio de despolitizar los medios y ejercer en libertad, con mayor base meritocrática y menor peso clientelar. Sin embargo, fue casi como un espejismo y los últimos años han visto un claro retroceso en este proceso de despolitización. Como suele suceder, los cambios tienen mayor repercusión cuando se refieren a la televisión pero acaban afectando a los medios en general.

Sea cual sea el signo político del partido en el poder, las radios con otro nombre (alternativas, culturales, educativas) nunca han encontrado una salida digna a una situación que dura demasiado. Se las tolera, como si de hacer un favor se tratara, mientras sirven de tapadera ideal de emisoras comerciales sin licencia, aunque, al final, las radios «legales» las incluyan todas bajo el epígrafe «piratas». Como apunta Clua (2011), la normativa vigente no está pensada para proyectos de base social que no acaben de encajar en la definición legal de medios sin ánimo de lucro, pero sí lo está para asociaciones o entidades que «difícilment podrien considerar-se de base ciutadana, com ara, posem per cas, associacions 0 fundacions promogudes per empreses, entitats financeres, institucions religioses, etc.» (p. 37). La no profesionalización de la producción cultural, las estructuras asamblearias o incluso la posibilidad de ocupar libremente el espectro radioeléctrico son algunos de los ejemplos que la autora apunta al referirse a la dificultad de encajar legalidad y medios que están fuera del mainstream. Por si fuera poco, como recuerda Zallo (2010), la Ley de 2010, a pesar de que el artículo 32 habla de ellas, las sitúa en el mismo plano que cualquier empresa que opte a una licencia pero las obliga a justificar el origen de sus fondos, algo que el autor califica, con razón, de «insultante sospecha».

En cuanto al marco normativo y la tecnología, la industria radiofónica española afrontó la digitalización de sus ondas en 1997 con el gobierno del Partido Popular (PP) a través de la Disposición Adicional 44a de la Ley 66/1997, de 30 de diciembre, de Medidas Fiscales, Administrativas y del Orden Social, más conocida como «Ley de Acompañamiento a los Presupuestos Generales del Estado». España adoptó oficialmente el estándar Eureka 147 (conocido popularmente como DAB) a través de dos artículos del Plan Técnico Nacional de la Radiodifusión Sonora Digital Terrenal (Real Decreto 1287/1999, de 23 de junio), dentro del cual también se planificó el espectro disponible para la radio digital de coberturas estatal y autonómica. Las emisiones de cobertura comarcal y supracomarcal se planificaron posteriormente (Orden de 15 de octubre de 2001) y las de ámbito local no sólo no se llegaron a planificar sino que se subastaron a principios de 2016 para su uso en downlink, es decir para la transmisión de información en enlace descendente.

Como es bien sabido, el fracaso de la radio digital en España, como en otros países, junto con la crisis económica, motivó algunos cambios en la normativa pero no detuvo la convocatoria de concursos para obtener licencias de radio en FM: 352 en 1989, 350 en 1997, y 866 en 2006 . A partir de entonces, la atención se focalizó en el apagón analógico de la televisión, que tuvo lugar el 3 de abril de 2010. Ese mismo año, en la Ley 7/2010 General de la Comunicación Audiovisual, la disposición transitoria decimoquinta dedicó dos artículos a la radio digital: en uno el gobierno se comprometía a elaborar un nuevo plan técnico de digitalización «integral», el segundo artículo prometía reunir a todos los sectores implicados. Un año después, el Real Decreto 802/2011 modificó dos aspectos del anterior plan técnico de 1999: a) las especificaciones técnicas que debían tener los transmisores y b) las fases de cobertura a las que, por ley, están obligados los radiodifusores, tanto públicos como privados.

Este Real Decreto permitió que se mantuviera el statu quo y enviaba el DAB a una especie de limbo tecnológico, ya que en su Artículo 4.1. afirmaba que el estándar para ese plan «integral» debería ser elegido y acordado por los radiodifusores (siempre de acuerdo a una norma ETSI). Concretamente, el acuerdo debía ser entre la entidades habilitadas de este servicio. Pero en el siguiente punto 2 se decía que, en caso de que no hubiera acuerdo, «los transmisores de las estaciones de radiodifusión sonora digital terrestre serán conformes a la norma de telecomunicaciones EN 300401 del Instituto Europeo de Normas de Telecomunicación (ETSI)». Es decir, DAB, ningún otro estándar tecnológico referido a audio digital de los existentes hasta entonces (DRM, Digital Radio Mondiale, IBOC, In-Band On-Channel, etc.), ni tan siquiera el DAB+. Al no existir una comunicación oficial, tal y como se exige, se sobreentiende que los radiodifusores están de acuerdo en continuar con el DAB. Aunque esto, por ahora, no tiene la más mínima importancia. Recordemos que según el estándar elegido, digitalizar el medio radio puede implicar un cambio de paradigma, es decir, un cambio de las estructuras industrial, económica, social de uso y consumo y política que forman parte del marco que sustenta una ventana de oportunidad para cualquier tecnología (Bonet et al., 2009).

\section{Estructura empresarial y programación}

La radio española sigue siendo, sustancialmente, la misma desde que el oligopolio quedó asentado a finales de los años 80 y principios de los 90. Tal y como en su momento ya se dijo, el mercado español no daba para más de tres grandes cadenas estatales privadas y así sucedió cuando la SER compró Antena 3 Radio. Desde entonces, el sector privado pivota alrededor de esta cadena, la COPE y Onda Cero, unas pocas cadenas privadas regionales, todo el abanico de oferta pública estatal, autonómica y local, así como unas pocas emisoras locales independientes.

Las grandes cadenas dependen de grupos de comunicación de talla considerable con intereses en otros medios e industrias culturales (especialmente televisión) y con accionistas de referencia que poco o nada tienen que ver con la comunicación (bancos, cajas, fondos de 
inversión, empresas del sector de la electricidad, la construcción, etc.). Por lo tanto, empresarialmente la radio española sigue más o menos igual, lo realmente novedoso es la miríada de pequeñas empresas de nuevo cuño, departamentos de nueva creación dentro de las empresas y páginas web con todo tipo de contenido en audio que han ido surgiendo dentro de o a su alrededor. El audio, como matriz de creación de contenidos, ya no es exclusivo de la radio tradicional. Esas nuevas empresas, departamentos y contenidos dotan de mayor dinamismo a un mercado que siempre se mostró más tradicional y estático que el de la televisión, convirtiendo a su vez la red en una suerte de feria o plaza de exhibición de contenidos para quien desee contratarlos o comprarlos. La red ha permitido mostrar su capacidad a mucha gente que de otro modo lo hubiera tenido más difícil, si bien esta democratización obliga a ser más exigentes en el posicionamiento (Sellas y Bonet, 2016).

Según el último resumen general del EGM (AIMC, 2016), correspondiente al período de octubre de 2015 a mayo de 2016, la radio española tiene una penetración del 60,4\%, por detrás de internet y la televisión. Con poca diferencia, es el tanto por ciento que ha venido manteniendo desde 2012, cuando empezó a remontar unos índices de penetración que no superaban el 58 \% desde 1997. Por día y de lunes a domingo, la programación generalista tiene un total de oyentes de 11.344, mientras que la temática tiene 14.682 (correspondiendo 1.105 a la temática informativa). Encabezan la lista de emisoras más escuchadas que ofrecen programación generalista la SER, la cadena COPE, Onda Cero y RNE, entre las cuatro representan el $76.1 \%$ del share. En esta lista de 11 emisoras, tienen cabida radios autonómicas de Cataluña (RAC1, privada, y Catalunya Ràdio, pública), Andalucía (Canal Sur), País Vasco (Radio Euskadi y Euskadi Irratia) y Galicia (Radio Galega). Más de la mitad de las emisoras más escuchadas son de cobertura autonómica, pero representan el $15 \%$ del share. En cuanto a la radio temática, de una lista de 23 , las primeras son Cadena 40, Dial, Europa FM y Cadena 100, es decir, las emisoras musicales de los grupos donde están también las cadenas que lideran la antena convencional (sumando un $48 \%$ del share).

Al igual que su estructura empresarial y a pesar de que siempre ha dado buenas muestras de creatividad, la programación de la radio española es bastante conservadora. Lo que se ha contado hasta este punto sobre tecnología, estructura y marco normativo sirve igualmente para la parrilla programática, todavía en pie pero sometida a experimentos de fragmentación y búsqueda y fidelización de la audiencia más joven, como variados estudios y artículos han demostrado hasta ahora (Gutiérrez et al., 2012 y 2014; Pedrero et al., 2015; Soengas, 2013).

\section{Financiación}

La financiación, como la programación y la cadena de valor en general, ha sufrido algunos cambios durante los últimos años por la digitalización y la aparición de internet como plataforma de distribución. Sin embargo, sus embites no se han hecho notar con igual fuerza que en otras industrias culturales, como la prensa, el cine, la música o la televisión.

Tabla 1. Evolución de los ingresos de la radio

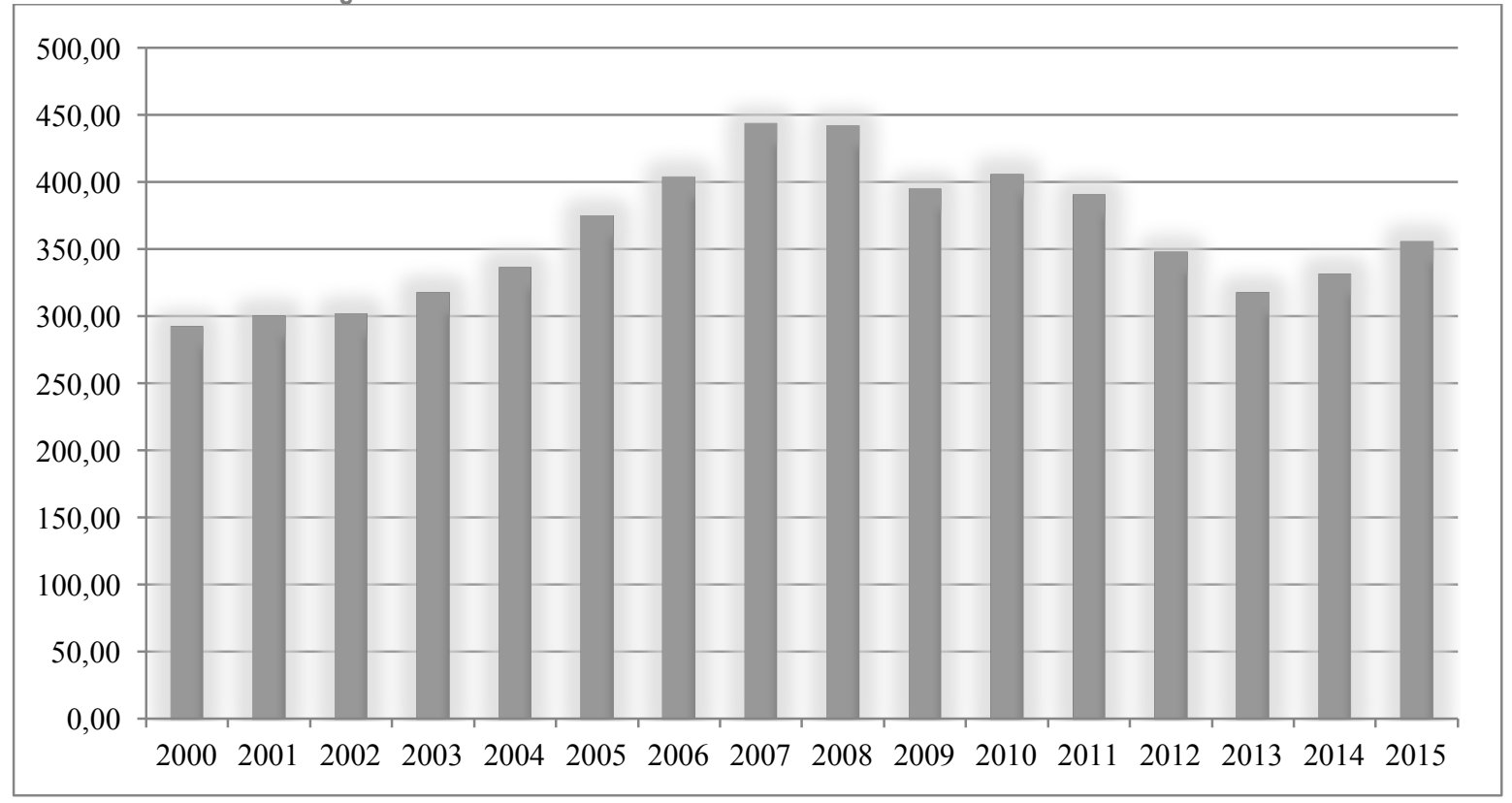

Fuente: elaboración propia a partir de los datos del Informe Anual 2016 de la CNMC.

Cuando en 2010, entrando ya en vigor la Ley 8/2009, de 5 de junio, de la Radio y la Televisión de Titularidad Estatal, Televisión Española dejaba de obtener financiación gracias a los ingresos por publicidad, hacía más de 20 años que había sucedido otro tanto con la radio pública. Recordemos brevemente que en 1991, como resultado del Plan de Implantación Territorial, que supuso el desmantelamiento de una parte de la red de Radio 5 y toda Radio 4, excepto, temporalmente, las emisoras de Sevilla y Barcelona, se consiguió ahorrar 1.800 millones, pero 120 trabajadores fueron despedidos, además de reubicados 154 fijos en otras emisoras de RNE, ocupando plazas vacantes por baja 0 jubilación anticipada y liberando algunas que estaban cubiertas por personal temporal. Al no mejorar la situación, en 1993 se firmó un contrato-programa con el Gobierno, mediante el cual el Ministerio de Economía y Hacienda subvencionaba a RNE, la Orquesta y Coros de RTVE y el Instituto de RTVE con 31.804 millones de pesetas para el ejercicio del mismo año y contemplaba incluir 31.000 millones dentro de los Presupuestos Generales de 1994 para compensar las pérdidas de 1992. El contrato supuso para RNE tener que depender desde entonces de la asignación establecida por el contrato-programa y perder la única fuente de ingresos publicitaria, dado que desde el día 1 de julio de ese año Radio 5 ya no podía admitir publicidad, para evitar la doble financiación (Ortiz Sobrino, 2012). 
A partir de ese momento, la radio española quedó dividida entre una radio pública estatal que no admitía publicidad y el resto de emisoras públicas y privadas que sí.

Siendo como es la principal fuente de ingresos, la publicidad no ha sido ni es la única. Las emisoras públicas reciben también asignaciones del erario público, algunas privadas ensayaron fórmulas como cotizar en Bolsa (Antena 3) o, ya en la línea de las radios alternativas, depender en gran medida, si no exclusivamente, de las cuotas de asociados, conciertos, rifas, etc. La derivada de estas opciones en internet serían las donaciones, el micromecenazgo, servicios premium, el patrocinio y otras que todavía están en fase de prueba / error. La versión digital de los medios necesita sostenerse de alguna manera y, a pesar de los cambios, acaba necesitando de un sistema de medición que permita poner en valor material el trabajo intelectual (Sellas y Bonet, 2016).

\section{Discusión y conclusiones}

No hay un solo momento en la historia de la radio que no pueda ser tomado como ejemplo de construcción social de una tecnología: su nacimiento como medio punto a punto, su transformación en un medio de comunicación de masas, su uso y disfrute en tiempos de paz y de guerra, la adaptación y competencia tras la aparición de nuevos medios, nuevas tecnologías, nuevos estándares. El caso español, además, ejemplifica a la perfección, con el DAB, la necesidad de estudiar y conocer las causas subyacentes al fracaso de una tecnología, a pesar de su óptima factura técnica.

La permanente capacidad de adaptación de la radio como medio puede hacer pensar en un movimiento continuo de cambio de alcance pero lo cierto es que hace años que, en esencia, la radio española es la misma: lo es en cuanto a estructura, lo es en cuanto a programación, audiencia y forma de financiación. Normativamente, no ha cambiado mucho y aunque parezca que hay un gran dinamismo, aunque éste existiera, se vería constantemente frenado porque la estructura de base, los pilares que sostienen todo el sistema no sólo no avanzan a la misma velocidad sino que, además, retroceden.

Todo ello no significa que la radio española no esté mutando, quiere decir simplemente que hay que dar tiempo y estar muy vigilantes a todo intento de que lo que está viviendo ahora no dependa tanto del pasado (path-dependence) y estén implicados en ello el máximo número de colectivos. La llamada radio 2.0 o 3.0, el entorno multipantalla o multiplataforma, el cross-media, son todo términos que ayudan a explicar qué está sucediendo pero a menudo son usados con excesiva generosidad. Dado que la radio ha dado siempre muestras de una gran capacidad de adaptación cada vez que alguien predijo su muerte (y no han sido pocas), lo realmente interesante es distinguir entre una nueva fase de adaptación y lo que de verdad está agitando lo que se conoce como core business, la esencia de su comportamiento y definición como industria cultural. Y algunos de los movimientos que empiezan a dar cuenta de cambios más profundos serían una mayor presencia de productores y de distinto tipo, una mayor variedad también de puntos de difusión, o una mayor dispersión y nivel de exigencia de los oyentes, así como

Pero la radio española sigue disponiendo de unas licencias de radio digital que no utiliza (irónicamente, ello evita que sean subastadas), lleva años sin dar muestras de querer dar un paso adelante y lo que ha hecho hasta el momento en tema de difusión digital es apuntarse a una plataforma que ya existe, Internet. EI DAB no es una panacea pero los países que han invertido más tiempo y dinero en él se han visto obligados a hacer gala de una mayor innovación y especialización programática y a buscar audiencias no servidas. Y ello es válido tanto para la radio pública como para la privada.

La radio española del futuro será lo que los colectivos más directamente implicados (productores, oyentes, difusores, empresarios, técnicos, políticos, etc.) quieran que sea. Así lo demostraron, en España y otros países, en el caso de la radio digital vía hertziana. El determinismo tecnológico es un buen anestésico que impide tomar consciencia de la necesidad constante de cuestionamiento y de la capacidad que la sociedad puede llegar a demostrar, en lo que a tecnologías de la comunicación se refiere.

Como algunos radiodifusores, tradicionales y de nuevo cuño, apuntaban en estudios sobre radio en Europa y España (Bonet et al., 2012; EBU, 2014, 2016; Sellas y Bonet, 2016), la clave reside en competir en contenidos, pero colaborar en tecnología, es decir, conocer la tecnología pero concentrarse e invertir en los contenidos. Al fin y al cabo, la digitalización, como proceso tecnológico general y de enorme alcance, ha sido la excusa o herramienta que una generación, los jóvenes, ha utilizado para recordarnos que los contenidos de la radio pueden ser de otra manera 0 quedarse como están, a riesgo de perderles como oyentes.

\section{Bibliografía}

Aibar Puentes, E. (2001). Fatalismo y tecnología: ¿es autónomo el desarrollo tecnológico? http://www.uoc.edu/web/esp/art/uoc/0107026/aibar.html Ala-Fossi, M. (2005). Mapping the technological landscape of radio: where do we go next?. First European Communication Conference. Amsterdam, 24-26 de novembre. CD-ROM.

Ala-Fossi, M., Jauert, P., Lax, S., Nyre, L., Shaw, H. (Eds.) (2010). Digital Radio in Europe. Technologies, industries and cultures. Bristol: Intellect. Ala-Fossi, M., Lax, S., O'Neill, B., Jauert, P., Shaw, H. (2008). The Future of Radio is Still Digital-But Which One? Expert Perspectives and Future Scenarios for Radio Media in 2015. Journal of Radio \& Audio Media, 15(1), 4-25.

AIMC (2016) Resumen General. Disponible en: http://www.aimc.es/-Datos-EGM-Resumen-General-html.

Arboledas, L. (2012). Regulación y políticas públicas: dispersión legislativa y clientelismo político. En: J. I. Gallego Pérez \& Ma. T. García Leiva (Coords.), Sintonizando el futuro: Radio y producción sonora en el siglo XXI (pp. 105-141). Madrid: Instituto RTVE.

Arboledas, L. y Bonet, M. (2014). The Radio in Spain: European Appearance, Franco«s Legacy. Javnost - The Public, 21(4), 67-82.

Bijker, W.E. (1995). Sociohistorical Technology Studies. En: S. Jasanoff, G. E. Markle, J. C. Petersen y T. Pinch (Eds.), Handbook of Science and Technology Studies (pp. 229-256). Thousand Oaks: Sage.

Bijker, W., Hughes, T. P., Pinch, T. (Eds.) (2012) anniversary edition. The Social Construction of Technological Systems. New Directions in the Sociology and History of Technology. Cambridge: MIT Press.

Bonet, M. (2005). La radio española 1994-2004. Una década de consolidación y desencanto. Sphera Pública, 5, 59-70. 
Bonet, M., Arboledas, L. (2011). The European exception: Historical evolution of Spanish radio as a cultural industry. Media International Australia incorporating Culture and Policy Journal, 141, 38-48.

Bonet, M., Arboledas, L., Guimerà, J. À. (2014). Past boundaries, future limitations: Spanish public service broadcasting at risk. Paper presentado en el RIPE@2014, celebrado en Tokyo del 27 al 29 de agosto. Disponible en: http://ripeat.org/library/Bonet\%20et\%20al\%20RIPE\%20paper\%202014.pdf.

Bonet, M.; Civil i Serra, M.; Llinés, M. (2008). Una década de políticas de gestión del espectro radioeléctrico en la Unión Europea (1997-2007). Análisis de las consultas públicas, el marco normativo y las prioridades estratégicas. Observatorio (OBS*) Journal, 7, 41-61. Disponible en: http://www.obs.obercom.pt/index.php/obs/article/view/170/222.

Bonet, M.; Corominas, M.; Fernández Alonso, I.; Díez, M. (2009). Keys to the Failure of DAB in Spain. Journal of Radio \& Audio Media, 16(1), 83101.

Bonet, M., Fernández-Quijada, D., Suárez Candel, R., Arboledas, L. (2012). Servei públic i innovació tecnològica. Informe final per al Consell de l«Audiovisual de Catalunya. Disponible en: https://www.cac.cat/pfw_files/cma/recerca/estudis_recerca/Servei_public_i_innovacio_tecnologica.pdf. Bonet, M., Guimerà i Orts, J. À. (2016). When efficiency meets clientelism: Spectrum management policy and broadcasting model - The Spanish case. European Journal of Communication, 31(4), 411-425.

Clua, A. (2011). Els mitjans comunitaris: la lluita i el reconeixement de les ràdios i les televisions de base social. En: M. de Moragas, M. Civil i Serra, I. Fernández Alonso, J. J. Blasco Gil y B. López (Eds.), Informe de la comunicació a Catalunya 2009 - 2010 (pp. 31-38). Barcelona: Generalitat de Catalunya.

David, P.A. (2007). Path dependence. A Foundational Concept for Historial Social Science. Cliometrica, 1(2), 91-114.

Fernández-Quijada, D. (2011). Medi@TIC. Anàlisi de casos de tecnologia i mitjans. Barcelona: Editorial UOC.

EBU (2014). Digital Radio Toolkit. Key Factors in the Deployment of Digital Radio. Disponible en: http://www.ebu.ch/files/live/sites/ebu/files/Publications/EBU-MIS_Digital-Radio-Toolkit.pdf

EBU (2016). Digital Radio 2016. Market Report. Disponible en: https://www.ebu.ch/files/live/sites/ebu/files/Publications/EBU-MIS - Digital Radio Report 2016.pdf.

Gutiérrez, M.; Ribes, X. Monclús, B. (2012). Percepción de los jóvenes ante las estrategias programáticas de la radio musical de antena convencional y online. El caso de Cataluña. Actas del III Congrés Internacional de l'Associació Espanyola d'Investigació de la Comunicació. Disponible en: http://www.ae-ic.org/tarragona2012/contents/comunicacions_cd/ok/210.pdf.

Gutiérrez, M.; Martí, J. M.; Ferrer, I.; Monclús, B.; Ribes, X. (2014). Los programas radiofónicos españoles de prime time en Facebook y Twitter: Sinergias entre la radio convencional y las redes sociales. Revista Latina de Comunicación Social, 69, 418- 434. Disponible en: http://www.revistalatinacs.org/069/paper/1018 UAB/21mes.html.

Hughes, T.P. (1989). The Evolution of Large Technological Systems. En: W. Bijker, T.P. Hughes y T. Pinch (Eds.), The Social Construction of Technological Systems. New Directions in the Sociology and History of Technology (pp. 51-82). Cambridge: MIT Press.

Lievowitz, S., Margolis, S. (2000). Path Dependence. En: B. Bouckaert \& G. De Geest (Eds.), Encyclopedia of Law and Economics, Volume I. The History and Methodology of Law and Economics (pp. 981-998). Cheltenham: Edward Elgar.

Lievrouw, L. A. (2006). Determination and Contingency in New Media Development: Diffusion of Innovations and Social Shaping of Technology Perspectives. En: L.A. Lievrouw y S. Livingstone (Eds.) Handbook of New Media. Social Shaping and Consequences of ICTs. London: Sage.

Mahoney, J. (2000). Path Dependence in Historial Sociology. Theory and Society, 29(4), 507-548.

McKenzie, D., Wajcman, J. (Eds.) (1999). The Social Shaping of Technology. 2nd. ed. Buckingham: Open University Press.

Ortiz Sobrino, M. A. (2012). Evolución del modelo de financiación de Radio 5. Del estatuto de radio y televisión de 1980 a la Ley general de comunicación audiovisual de 2010. Icono 14. Revista de comunicación y tecnologías emergentes, 10(3), 383-402.

Page, Scott E. (2006). Path Dependence. Quarterly Journal of Political Science, 2006, 1, 87-115.

Pedrero, L. M.; Sánchez Serrano, C.; Pérez Maílo, A. (2015). Desafíos de la industria radiofónica española en el entorno digital: hacia la redefinición de su modelo de producción, comercialización y difusión. En: El nuevo diálogo social: organizaciones, públicos y ciudadanos. Valencia: Campgràfic. Disponible en: http://forodecomunicacion.org/wp-content/uploads/2016/04/Foro2014.pdf.

Pinch, T., Bijker, W. (1989). The Social Construction of Facts and Artifacts: Or How the Sociology of Science and the Sociology of Technology Might Benefit Each Other. En: W. Bijker, T.P. Hughes, y T. Pinch (Eds.) The Social Construction of Technological Systems. New Directions in the Sociology and History of Technology (pp. 11-44). Cambridge: MIT Press.

Piñeiro Otero, T. (2015). Los 'Radio Studies' en España. Tres décadas de investigación en las revistas académicas de Comunicación. Estudios sobre el Mensaje Periodístico, 21(2), 1169-1188.

Porter, M. (1998). $2^{\text {nd }}$ ed. Competitive Advantage. Creating and Sustaining Superior Performance. New York: The Free Press.

Rogers, E.M. (2003). Diffusion of innovations, 5th. ed. New York: Free Press.

Sellas, T. (2013). La ràdio a les xarxes socials. Anàlisi de l«ús, dinàmica i motivacions de la presència de les emissores generalistes a Facebook i Twitter. Informe per al Consell de l'Audiovisual de Catalunya Disponible en: http://www.cac.cat/pfw_files/cma/recerca/estudis_recerca/Radio_a_les_xarxes_socials_TSellas.pdf

Sellas, T., Bonet, M. (2016). L'àudio a Internet: de la ràdio en línia als projectes nadius. Informe final per al Consell de l'Audiovisual de Catalunya. Soengas, X. (2013). Retos de la radio en los escenarios de la convergencia digital. adComunica. Revista de Estrategias, Tendencias e Innovación en Comunicación, 5, 23-36.

Tosoni, S. with Trevor Pinch (2016). Entanglements. Conversations on the Human Traces of Science, Technology, and Sound. Cambridge: MIT Press.

Williams, R. y Edge, D. (1996). The social shaping of technology. Research Policy, 25, 865-899.

Winner, L. (1999). Do artifacts have politics? En: D. MacKenzie y J. Wajcman (Eds.) The Social Shaping of Technology (pp. 28-49). 2nd. ed. Buckingham: Open University Press.

Winston, B. (1998). Media Technology and Society. A History: from the Telegraph to the Internet. London: Routledge.

Zallo, R. (2010). La política de Comunicación Audiovisual del gobierno socialista (2004-2009): un giro neoliberal. Revista Latina de Comunicación Social, 65, 14-29. http://www.revistalatinacs.org/10/art/880_UPV/02_Zallo.html 
BONET-BAGANT, M. (2017): “¿Qué hay de nuevo, radio? Claves para un análisis sobre el futuro de la radio española desde el constructivismo social de la tecnología". En Revista de la Asociación Española de Investigación de la Comunicación, vol. 4, número 7, pp. 14-21. 